
\title{
28 Research Square \\ Comparison of Pancreatic Cancer Treatment Outcomes: A Real-World Data Analysis
}

Jacob Vikström ( $\square$ vikstrom.jacob@outlook.com )

University of Helsinki https://orcid.org/0000-0002-8668-7944

Georgii Shangin

Vaasan keskussairaala

Tommi Viitanen

2M-IT

\section{Natalja Eigeliene}

Vaasan keskussairaala

Antti Jekunen

Vaasan keskussairaala

\section{Research article}

Keywords: Pancreatic adenocarcinoma, gemcitabine, nab-paclitaxel (Abraxane), real-world data, adverse effect

Posted Date: September 15th, 2020

DOl: https://doi.org/10.21203/rs.3.rs-65437/v1

License: (c) (i) This work is licensed under a Creative Commons Attribution 4.0 International License. Read Full License

Version of Record: A version of this preprint was published at Pancreas on February 1st, 2022. See the published version at https://doi.org/10.1097/MPA.0000000000001990. 


\section{Abstract \\ Background}

Since the ground-breaking phase III MPACT trial showed clear benefit of gemcitabine-nab-paclitaxel, this regimen has emerged as the standard of care for advanced pancreatic adenocarcinoma (PAC). Prior to this study, few studies have shown how results from randomized controlled trial translate to the real world. This study investigated how patients fared in the real world.

\section{Methods}

This single-centre, retrospective study was conducted in the Vaasa Central Hospital, Finland. 148 patients with PAC (ICD-10 C25) between 1/2011-12/2016 were identified with resectable, locally advanced or metastatic disease. Information about the basic characteristics, treatment regimens and adverse effects (AEs) were extracted from patient files and analysed.

\section{Results}

The median overall survival (OS) was 10.4 months for treated and 2.8 months for untreated patients. For metastatic disease OS was 4.9 months, for locally advanced 9.1 months. Patients who received 3 or more treatment lines had OS 19.1 months, while those who received 2 or 1 line had 11.3 and 6.2 months, respectively. The following grade $2-4$ haematologic toxicities occurred: anaemia $(n=19)$, leukopenia $(n=$ $49)$, neutropenia $(n=55)$ and thrombocytopenia $(n=9)$. Febrile neutropenia occurred 7 times. A total of 12 grade 5 AEs were recorded in 7 patients, and 3 other patients died of unknown complications.

\section{Conclusion}

This study shows that active treatment is worth pursuing in most PAC patients and study results from this real-world data study differ from randomized controlled trials. Special caution should be applied when continuing chemotherapy in patients with ECOG 2 and who are older than 70 years.

\section{Background}

The diagnosis of pancreatic ductal adenocarcinoma (PAC) has been associated with poor outcomes in the vast majority of patients since it was first identified. Although the mortality rates associated with the majority of cancers are falling, the incidence of PAC is increasing, and its associated mortality rate remains the same as it was a decade ago; the 5 -year survival rate is $2-5 \%(1-3)$. Pancreatic cancer (PC) was the third most common cause of cancer-related death in Finland between 2013 and 2017(1), and globally, it was the seventh most common in 2015 (2). These poor outcomes might be partly explained by the fact that PAC is an aggressive cancer that silently progresses, and the majority of patients are 
diagnosed with stage IV metastatic disease (3). At this late stage, surgical intervention with curative intent is rarely possible, and few treatment options other than non-curative chemotherapy remain (4).

Gemcitabine (gem) monotherapy has been the standard first-line treatment for PAC since Burris et al. in 1997 showed significantly better results with the newly approved gem than with the traditionally used 5fluorouracil (5-FU)(5). Subsequent trials over the past two decades have unsuccessfully explored the use of gem-combination therapies, trying to increase the OS when compared with the results obtained with gem monotherapy (6-11).

Only recently in the phase III MPACT and PRODIGE4/ACCORD 11 studies have the use of combination regimens such as oxaliplatin, irinotecan and 5-FU/leucovorin (FOLFIRINOX) and gem plus nab-paclitaxel (gem + nab-P) been shown to have superior efficacy to gem monotherapy $(12,13)$. These combination regimens resulted in improvements in the median OS and in disease-related symptoms (median OS $6.8 \mathrm{vs}$ 11.1 months, $p<0.0001$ for gem vs FOLFIRINOX and 6.7 vs 8.5 months $p<0.00015$ for gem vs gem + nab-P) (14). Currently, both FOLFIRINOX and gem + nab-P are used as "gold standard" first-line (1L) treatments rather than gem monotherapy for PAC and especially for metastatic PAC (15). However, both of these treatment regimens are associated with significantly higher levels of toxicity, and therefore, they are recommended only for patients with Eastern Cooperative Oncology Group (ECOG) performance status scores of $0-1$ (16). In cases in which neither FOLFIRINOX nor gem + nab-P can be used because the patient has a ECOG score of 2 or higher or because of the risk of grades 3-4 AEs, gem monotherapy is still the preferred treatment (16). To date, a few studies have developed treatment options following the progression of disease after $1 \mathrm{~L}$ treatment (17-27), but a reliable standard second-line (2L) or later-line treatment has yet to be established; currently, many different regimens are being considered and offered $(14-16,20)$.

Most of the studies that have been conducted in search of a new standard treatment for PAC have been set up as clinical trials, with very little real-world data (RWD) used for comparison or verification $(28,29)$. Randomized controlled trials (RCTs) have long been considered the "gold standard" for obtaining clinical data on the efficacy and safety of treatments(30), but they are expensive and take a relatively long time to obtain results that could be utilized in regular clinical practice. RCTs are strictly controlled by a protocol designed to determine whether there is a beneficial treatment effect in a selected patient population. Usually, treatments before the initiation of a protocol are highly restricted, while no restrictions are usually made after explorative treatment. In the real-world setting, it is challenging to recreate the same conditions, and therefore, RCT evidence is used to treat patients who are not actually represented in primary RCT study populations.

Recently, however, discussions about the potential role of using RWD or real-world evidence (RWE) to provide additional information that could improve health outcomes rather than relying on RCTs alone have started to take place (31). RWD from regular clinical practice better reflect the reality of treatment usage and the effects on patient outcomes, as the treatments administered to patients are not dictated by a protocol intended to address certain research questions. Treatment decisions are made by individual 
doctors and are influenced by patients, relatives, hospital regulations, other social factors and the financial situation of the patients. Thus, it is likely to become increasingly important to ensure that treatment regimens truly provide benefits when used as a part of routine clinical practice in real populations, not just in RCTs (32). Comparisons of RWD and data from clinical trials serve as an important part of adapting new treatments for use in clinical practice and increase transparency between clinicians and those developing the treatment regimens; this process, in turn, is likely to help individual patients receive the right treatment at the right time $(30,33)$.

In the present study, we conducted a retrospective analysis with RWD from Vaasa Central Hospital to evaluate the effects of various treatments on outcomes in patients with PAC, spanning the spectrum from no treatment to a maximum of seven lines.

\section{Materials And Methods}

\section{Patients}

Patients who were diagnosed with pancreatic cancer (PC), specifically PAC, and admitted to the oncology clinic of Vasa Central Hospital between January 2011 and December 2016 were included in this retrospective study. First, a search was carried out in a patient register by a statistician using the ICD-10 code for PAC (C25). The list of eligible patients was extracted, and 160 candidates who met the criteria were identified $(n=160)$. (Fig. 1A). Then, two independent researchers in 2018-2019 carefully reviewed the patient files to confirm the diagnosis based on the histopathology reports of biopsies or postsurgical material. Patients who were diagnosed with a neuroendocrine tumour (NET) were excluded $(n=10)$. Additionally, patients who were initially clinically diagnosed with PAC and started chemotherapy for this indication but were later found to have a different primary tumour (such as metastasized NSCLC or cholangiocarcinoma) were excluded $(n=2)($ Fig. 1A).

Information such as patient age, sex, tumour characteristics (resectable, locally advanced or metastatic), operation status, comorbidities, baseline biochemistry test results (alanine aminotransferase (ALAT) and bilirubin levels and leukocyte and neutrophil counts), carbohydrate antigen 19 - 9 (CA19-9) levels, treatment regimens, performance status, hospitalization and number of chemotherapy treatment lines received was extracted from the patient files. A treatment line was defined as a minimum of 3 cycles of the same chemotherapy regimen. The primary endpoints were overall survival (OS), the frequency and grade of adverse effects (AEs), type of treatment and number of treatment lines received.

\section{Statistical Analyses}

Patient and tumour characteristics, treatments and AEs are reported as frequencies and percentages for categorical variables and median with ranges or interquartile ranges or means with $95 \%$ confidence intervals for continuous variables. These characteristics are also reported in the subgroups. 
Kaplan-Meier survival analysis was used to investigate OS and determine differences in survival between the subgroups. Significant differences between the subgroups were determined with the log-rank test, and the results are presented as the survival curves. Analyses were performed using $\mathrm{R}$ version 3.5.3.

\section{Results}

\section{Patient characteristics}

A total of 148 patients who met the inclusion criteria were identified and included in the analysis (Fig. 1 a). Of these 148 patients, 109 had received chemotherapy as either curative neoadjuvant, adjuvant or first-line non-curative treatment. The tumour and patient characteristics are listed in Table 1. The patients were divided into subgroups according to the stage of disease at diagnosis, and their baseline characteristics were analysed. The male to female ratio in our study was 77:71. Age was determined from the date of birth to the date of diagnosis, and the median age was 70.5 years. The distribution of ECOG scores at baseline was as follows: 22 patients had an ECOG score of 1 (of whom 10 had locally advanced disease, 9 had metastatic disease, and 3 had resectable disease), 106 patients had an ECOG score of 2 (of whom 39 had locally advanced disease, 49 had metastatic disease, and 18 had resectable disease) and 20 patients had an ECOG 3 (of whom 5 had locally advanced disease and 15 had metastatic disease) (Table 1).

At baseline, the median biochemical values were $31 \mathrm{U} / \mathrm{I}$ for plasma ALAT (females/males: 27/31 in the locally advanced group, $32 / 30$ in the metastatic group and 45/38.5 in the resectable group), $13 \mu \mathrm{mol} / \mathrm{I}$ for plasma bilirubin (13/16 in the locally advanced group, 13/12 in the metastatic group and 13/15.5 in the resectable group) and $284 \mathrm{kU} / \mathrm{I}$ for serum CA19-9 (49/176 in the locally advanced group, 2194/1211.5 in the metastatic group and 22/15 in the resectable group). Fifty-two patients (35\%) had diabetes type I or type II (14 in the locally advanced group, 29 in the metastatic group and 9 in the resectable group). Ninety-four patients $(63.5 \%)$ were using opioids for pain management during our study period. A total of 35 patients (23.6\%) had major comorbid illnesses ( 26 in total) or secondary cancers ( 9 in total) before or during our study period, such as hypertension, ischaemic heart disease, kidney cancer, breast cancer, severe depression or brain infarction. (Table 1)

\section{Tumour characteristics}

The primary tumour was found in the head of the pancreas in 91 patients, in the body of the pancreas in 13 patients or in the tail of the pancreas or another undefined location in 35 patients (Table 1). The median tumour size was $3.7 \mathrm{~cm}(0.4-14 \mathrm{~cm})$. For males and females respectively, the tumour sizes were $3.5 \mathrm{~cm}$ and $4 \mathrm{~cm}$ in the locally advanced group, $3.4 \mathrm{~cm}$ and $4.5 \mathrm{~cm}$ in the metastatic group and $3.2 \mathrm{~cm}$ and $3.5 \mathrm{~cm}$ in the resectable group. The tumour stage at diagnosis was metastatic in $49.3 \%$ of the patients, locally advanced in $36.5 \%$ and resectable in $14.2 \%$ (Supp Fig. 5). 


\section{Treatment results}

For the patients who received treatment of any kind, the median OS was 10.4 months (8.6-13.3 95\% Cl), while the patients who did not receive any treatment had a median OS of 2.8 months $(2.2-5.295 \% \mathrm{Cl})$ $(p<0.01)$ (Fig. 2 a). Figure $2 \mathrm{~b}$ shows the $0 S$ for all patients according to treatment characteristics. Patients who received neoadjuvant or adjuvant chemotherapy after surgical intervention had a median OS of 31.2 months $(16.9-\mathrm{NA} 95 \% \mathrm{Cl})$, while patients who received chemotherapy as a $1 \mathrm{~L}$ treatment had a median OS of 8.6 months $(7.0-10.695 \% \mathrm{Cl})$, and the patients who received no treatment had a median OS of 2.8 months $(2.2-5.295 \% \mathrm{Cl})(\mathrm{p}<6 \mathrm{e}-10)$ (Fig. $2 \mathrm{~b})$. A total of 77 patients received chemotherapy as a $1 \mathrm{~L}$ treatment. Sixty-three patients received gem monotherapy, 7 received gem+nab-P, and 7 received some other regimen as the $1 \mathrm{~L}$. The group receiving gem monotherapy had a median OS of 7.6 months (6.6-9.8 $95 \% \mathrm{Cl}$ ), the group receiving gem+nab-P had an median OS of 10.6 months (6.3-NA $95 \% \mathrm{Cl}$ ), and the others group had a median OS of 18.2 months (9.2-NA 95\% Cl) $(p<0.107)$ (Fig. 3 a and Supp Fig. 6).

Figure $3 \mathrm{~b}$ shows OS according to the number of treatment lines given. Patients receiving 3 or more lines of chemotherapy regardless of the regimen had a median OS of 19.1 months $(16.8-33.195 \% \mathrm{Cl})$; this group was composed of 15 patients. The patients who received only one line of treatment had a median OS of 6.2 months $(4.9-8.295 \% \mathrm{Cl})$; this group was composed of 49 patients. Patients who received 2 lines of chemotherapy had a median OS of 11.3 months (10.2-NA 95\% Cl); this group was composed of 13 patients $(p<0.002)$ (Fig. 3 b).

OS in patients with metastatic PAC and locally advanced PAC is shown in Figure $2 \mathrm{c}$. Patients who were diagnosed with locally advanced disease had a median OS of 9.1 months $(7.3-15.895 \% \mathrm{Cl})$, whereas patients with metastatic disease had a median OS of 4.9 months $(3.8-7.595 \% \mathrm{Cl})$. $(\mathrm{p}<0.008)$ (Fig. $2 \mathrm{c}$ ).

To overcome the limitation of a small sample size and better assess the outcomes of the patients receiving gem+nab-P, Figure $4 \mathrm{a}$ and $\mathrm{b}$ show the OS of patients who received gem+nab-P at any time during treatment versus the other patients. Figure 4 a shows all patients, excluding patients who underwent surgery, while Figure $4 \mathrm{~b}$ shows only those with metastatic disease. The patients who received gem+nab-P had a median OS of 18.3 months $(11.2-31.995 \% \mathrm{Cl})$, while those who received some other type of treatment had a median OS of 7.2 months $(6.3-9.195 \% \mathrm{Cl})(\mathrm{p}<0.008)$ (Fig. 4 a). Among the patients with metastatic disease, those who received gem+nab-P had a median OS of 11.2 months (8.2NA $95 \% \mathrm{Cl}$ ), while those who received other types of treatment had a median OS of 6.3 months (4.4-9.5 $95 \% \mathrm{Cl})(\mathrm{p}<0.112)$ (Fig. 4 b).

\section{Safety}

The frequency, types and grades of AEs are listed by subgroup in Table 2. AE classification was performed according to version 4 of the National Cancer Institute Common Terminology Criteria for Adverse Effects (CTCAE). The most commonly observed side effects in this study were grades 2-4 bone marrow toxicities, with 19 cases of anemia, 49 cases of leukopenia, 55 cases of neutropenia and 9 cases 
of thrombocytopenia. Febrile neutropenia was observed 7 times. Other commonly observed AEs were nausea, diarrhoea and infections related to the administered treatment. In our study, a total of 12 fatal AEs were recorded, with 1 fatal case of thrombocytopenia, 3 cases of fatal sepsis, 1 case of fatal leukopenia, 3 cases of fatal haemorrhage, 1 case of fatal cholecystitis, 1 case of fatal diarrhoea and 2 cases of fatal febrile neutropenia. An important observation is that while a total of 12 fatal AEs were recorded, they occurred in a total of 7 patients, with a few patients experiencing more than one fatal $A E$ simultaneously. Five of the patients with fatal AEs had locally advanced disease (all of them were older than 70 years, and 4 of these had ECOG scores of 2, while 1 had an ECOG score of 1), 1 of the patients had metastatic disease (older than 70 years, with an ECOG score of 2) and 1 had resectable disease (aged 65, with an ECOG score of 2). The division by subgroup yielded the following results: out of all 281 recorded $A E s$ of any grade, 116 occurred in patients with metastatic disease, 110 in patients with locally advanced disease and 55 in patients with resectable disease. Another 3 patients died within one month of the administration of treatment; however, because these fatalities did not occur in the hospital setting and there was no indication of a direct treatment-related $A E$, they were excluded from the toxicology analyses. None of these 3 patients were receiving their first treatment cycle when they died.

AEs seen only in individual patients or rarely associated with chemotherapy were all grouped together in the Other group. (Table 2)

\section{Discussion}

To our knowledge, this study provides some of the most mature RWD about treatment outcomes from a single institute and serves as a report on how results from RCTs translate to patients in routine clinical practice.

Although the OS rate of PC patients is one of the worst in any disease, our data clearly show the benefit of active treatment. As seen in Fig. 2a, the treated group had a significantly longer OS than the untreated group. Active treatment results in several additional months of life. Extending the life expectancy from 2.8 months to 10.4 months (Fig. 2a) is significant, and it is important to discuss this possibility with every patient diagnosed with PAC. Furthermore, our analysis showed a clear benefit of surgery in patients with resectable disease and, to some extent, those with locally advanced disease. Patients who received neoadjuvant or adjuvant chemotherapy achieved a median OS of 31.2 months compared to 8.7 months for patients receiving $1 \mathrm{~L}$ chemotherapy and 2.7 months for untreated patients (Fig. $2 \mathrm{~b}$ ). While locally advanced disease is usually inoperable, a few of our patients with locally advanced disease underwent surgery. Our study also clearly showed the generally more favourable median OS of patients diagnosed with locally advanced disease compared with the OS of those diagnosed with metastatic disease (9.2 vs. 4.9 months) (Fig. 2c). This indicates the need for better diagnostic tools, such as biomarkers, and better imaging technology, to increase the number of patients who are diagnosed at an earlier stage of disease, as other studies have also shown (34-38); novel therapies are also needed. 
Increasing the treatment duration and number of treatment lines administered resulted in a significant increase in OS from 6.3 months with one line of treatment up to 19.1 months with 3 or more lines of treatment $(p<0.002)$ (Fig. 3b). This finding is subject to survival bias, with healthier patients living longer and therefore receiving more treatment. It is thus not necessarily the treatment that is prolonging their lives. Further research on the subject is needed before conclusions can be drawn. However, these results provide evidence of the benefit of treating younger patients with lower ECOG scores more aggressively for longer periods of time.

Even though our data largely show relatively short survival times after diagnosis, there were some individual cases that stood out. A few patients treated early during our study period were still alive after 5 years and showed no signs of disease, with one patient showing complete remission of disease (confirmed via radiological examination) after a single cycle of gem monotherapy (PAD was positive for PAC). While this outcome is rare and further research is needed, these cases may serve as a source of hope for researchers, clinicians and patients because they show that remission can occur.

Because the combination of gem + nab-P was approved for PAC rather late during our follow-up period, the number of our patients who received this combination was low. Seven patients received gem + nab-P as a $1 \mathrm{~L}$ treatment, while 63 patients received gem monotherapy, and 8 patients received another treatment. This limited our analysis of the efficacy and safety of this combination compared to the efficacy and safety of gem monotherapy.

To better assess the efficacy of gem + nab-P combination treatment, we analysed the OS of patients who received gem + nab-P at any time during their treatment schedule (among all treated patients and in the subgroup of patients with metastatic disease). The results were fairly conclusive; in all patients using gem + nab-P, the median OS was 18.3 months, which was longer than the 7.3 months in patients receiving other types of treatment, including gem monotherapy $(p<0.008)$. This analysis included all patients (except patients who had undergone surgical resection of the tumour) (Fig. 4a). In the subgroup of patients with metastatic disease, the results were also promising, with those who received gem + nab-P obtaining a median OS of 11.2 months, while those who received other treatments obtaining a median OS of 6.3 months; however, this difference lacked statistical significance $(p<0.112)$ (Fig. 4b).

Due to its poor toxicity profile and the older age and poor performance status of our population, no patients in our study received FOLFIRINOX. Our analysis shows that there were significant toxicities and safety concerns associated with the administered treatments. A total of 10 ( 7 with recorded AEs and 3 without known cause) patients died within 30 days of chemotherapy administration. The most commonly observed AEs were grades 2-4 bone marrow toxicities, nausea, diarrhoea and infections. The frequency of AEs was high, with almost every patient experiencing an $\mathrm{AE}$ and many patients experiencing more than one. Grades 2-5 AEs occurred most frequently in the metastatic subgroup (134 out of 281), closely followed by the locally advanced subgroup (86 patients).

Due to key similarities in patient characteristics and geographical location, we used the results from the 2019 study by Bloomberg et al. (29) as a reference and compared the results to our own. We also 
considered the results from the landmark MPACT study by Von Hoff et al. from 2013 (12) when analysing our own results. However, due to significant differences between the inclusion/exclusion criteria and the manner in which our study was completed, a direct comparison of results between the MPACT study and our retrospective report is not possible. The present study and the study by Blomstrand et al. included all diagnosed cases of PC, with $50.7 \%$ of our patients having locally advanced or resectable disease, while the MPACT study only included patients with confirmed metastatic disease who were relatively young and in good condition.

Bloomberg et al. performed analyses to compare their results with those of the MPACT trial. Their study showed a longer estimated median OS for patients with metastatic disease than the median OS observed in the MPACT trial (9.4 vs 8.9 months), while our cohort had an estimated median OS of only 4.9 months. However, our patients in the metastatic subgroup were significantly older and in worse condition than those included in the MPACT trial or the study by Blomstrand et al., with 36 out of 73 patients with metastatic disease older than 70 and 64 patients with an ECOG score of 2 or 3 . In the MPACT study, $58 \%$ of the patients were younger than 65 years and had a Karnofsky score $>60$, which is equivalent to an ECOG score of $0-2$. The MPACT study included only patients with metastatic disease, whereas we included all diagnosed patients. $(12,13)$

Our study showed a mildly improved survival for patients in all three subgroups who received $1 \mathrm{~L}$ chemotherapy (regardless of disease stage) compared with the MPACT trial. Our patients receiving gem monotherapy had a median OS of 7.7 months (vs MPACT 6.7 months), those receiving gem + nab-P had a median OS of 10.5 months (vs MPACT 8.5 months), and those receiving other therapies had a median OS of 18.5 months $(p<0.171)$. Our analysis was conducted with all patients receiving $1 \mathrm{~L}$ chemotherapy (not including patients who underwent surgical resection) due to the small patient population. Notably, however, we obtained very similar results in terms of OS when compared with the study by Blomstrand et al.; their patients had a median OS of 10.9 months compared to the OS of 10.5 months in our gem + nabP subgroup.

The results of the study by De Vita et al (39) and a meta-analysis of 50 studies presented at an international conference suggest that with careful and appropriate patient selection, a median OS of longer than 12 months can be attained with $1 \mathrm{~L}$ gem + nab-P (40).

The patients in the present study experienced AEs more frequently and with higher grades than those in the study by Blomstrand et al., the MPACT study and previous studies on the subject $(41,42)$. The most common side effects in the aforementioned studies were bone marrow toxicities. Since no data on dosage have been published, we cannot with confidence determine the cause of this discrepancy. Both of the studies used for comparison collected data from $1 \mathrm{~L}$ treatment only, while the present study included all treatment lines, with a large proportion of patients receiving 2 or more lines (36\%) and 15 patients receiving 3 or more lines. This increased exposure to therapy regimens and longer follow-up time coupled with advanced age and high ECOG scores might contribute to the higher numbers of AEs observed. 
While Blomstrand et al. published RWD, there are key differences in the design of our study. The primary difference is that they examined the efficacy of gem + nab-P combination therapy in a real-world population and compared their findings with the established MPACT study. Our study is mainly a retrospective report of the outcomes in all treated PAC patients in a hospital in central Finland during an approximately six-year period. However, our study provides important and mature data with substantial value to clinicians. The present study included every patient diagnosed with PAC regardless of insurance status, comorbidities, age, sex, ethnic subgroup, or socioeconomic status and therefore provides a very close approximation to the outcomes of treatment with many of the accepted therapies today in patients in Finland diagnosed with PAC.

The present study involved a heterogeneous patient group, which made analysis and interpretation of the results difficult and, to some extent, impossible. The heterogeneity and lack of standardization in $1 \mathrm{~L}$ treatment regimens was the most disruptive factor and is also the largest limitation of our study. Due to these limitations, direct evaluation of efficacy between individual treatment regimens was not feasible.

We believe that the treatment decisions made during our study period were influenced by an array of factors, including age and ECOG score at baseline, which could partly explain why a relatively small number (only 77 out of 148 patients) received chemotherapy as a $1 \mathrm{~L}$ treatment and only 7 patients initially received gem + nab-P combination therapy. Furthermore, the fact that change has been slow in the field of PAC treatment, with no new regimens introduced between 1997 and 2011/2013 that were improvements compared with gem monotherapy, together with the many years of clinical experience with the use of gem monotherapy, can provide an explanation for the low number of patients receiving $1 \mathrm{~L}$ combination therapy.

In our study, AEs frequently occurred during chemotherapy administration. The frequency was higher than that reported in the literature, mainly because our patients were older and had worse performance status scores. Seven patients died during treatment. Another three patients died without clear signs of disease progression; these deaths were registered as treatment-related deaths, but none of these patients died after the first chemotherapy cycle. This means that the initial chemotherapy was tolerated, but as treatment was continued, the level of toxicity became too high in some patients. Notably, among the patients who died during treatment, one patient who died had resectable disease, was 65 years old, and had an ECOG score of 2; thus, this patient should have had a more favourable prognosis and should have been treated actively. Another patient who died from AEs had metastatic disease, was older than 70 years and had an ECOG score of 2. Similarly, 5 other deaths occurred in patients with locally advanced disease, who were over 70 years old and, with the exception of 1 patient with an ECOG score of 1, had ECOG scores of 2. Overall, in our study population, 106 patients (72\%) had an ECOG score of 2 and a median age of 70.7 years, and $50 \%$ of the study patients were older than 70 years. Therefore, if active treatment had been denied to patients older than 70 years or who had ECOG scores of 2 or more, then most of our patients would not have had the chance to obtain the clear benefit they received from active treatment with chemotherapy. However, we think that performing more active follow-up during treatment and allowing temporary or permanent discontinuation of therapy might affect the occurrence in side effects 
and decrease the number of treatment-related deaths. In addition, based on our data, those patients who can tolerate it should continue therapy.

These findings strongly suggest the need for more tolerable therapies and more research to further establish patient selection criteria for every treatment regimen; treatments are especially needed for patients with ECOG scores of 2 and those with metastatic disease who are in good condition. Further research is also needed on protocols and patient selection criteria for the continuation of treatment beyond the $1 \mathrm{~L}$. This coupled with definitive protocols based on evidence regarding when and which patients should receive palliative care instead of continuing with active therapy could also save resources. Furthermore, this study clarifies the need for RWD to supplement RCTs. Analysis of RWD could reduce severe side effects and deaths caused by overtreatment, providing a reference for individual physicians when determining the best treatment for patients with PC.

In the last few years, the efficacy of $1 \mathrm{~L}$ FOLFIRINOX or gem + nab-P vs gem monotherapy for metastatic PAC has been widely investigated. Since no three-arm RCTs measuring efficacy have been conducted, real-world observations are important to inform decision making. Many studies have hinted that the OS achieved with treatment with FOLFIRINOX is slightly superior to that achieved with gem + nab-P (14.1 compared to 10.5 months according to Wang et al 2019 or 17.1 compared to 11.5 months according to Sasaki et al 2019) $(28,43)$. However, the same studies also concluded that FOLFIRINOX should be reserved for a very small portion of the patient population due to its toxicity profile. Aroldi $\mathrm{F}$ et al. proposed the following treatment algorithm for metastatic PAC: patients with a performance status score of $0-1$, who are younger than 65 years and who have no comorbidities can receive FOLFIRINOX as a $1 \mathrm{~L}$ treatment; patients with an ECOG score of $0-1$ who are older than 65 years should receive gem + nab-P as a $1 \mathrm{~L}$ therapy; and patients with an ECOG score $>1$, who have serious comorbidities and are older than 65 years should receive gem monotherapy as a $1 \mathrm{~L}$ treatment (18). After disease progression, Aroldi $\mathrm{F}$ et al. proposed that the $2 \mathrm{~L}$ treatment should depend on the $1 \mathrm{~L}$ treatment. Patients who received $1 \mathrm{~L}$ FOLFIRINOX should receive gem + nab-P or gem monotherapy, those who received $1 \mathrm{~L}$ gem + nab-P should receive either platinum-based therapy (Xelox, Gemox or OFF), irinotecan-based therapy (FOLFIRI, Nal-Iri, $\mathrm{Nal-Iri}+5$-FU-leucovorin) or fluoropyrimidine, and patients who received $1 \mathrm{~L} \mathrm{gem} \mathrm{monotherapy} \mathrm{should}$ receive irinotecan monotherapy or fluoropyrimidine after consideration of the condition of the patient (18). Most studies recommend $2 L$ therapy for patients who are able to tolerate it, with a few studies describing prognostic factors for receiving and benefiting from $2 L$ therapy $(23,25,26)$. For example, Vienot et al. (26) presented a prognostic model to predict which patients would benefit the most from $2 \mathrm{~L}$ therapy. Their study found that age, smoking status, liver metastases, ECOG performance status score, jaundice, duration of $1 \mathrm{~L}$ treatment, type of $1 \mathrm{~L}$ regimen and pain were prognostic factors. Pokataev et al. (23) provided another prognostic model to predict which patients would benefit the most from $2 \mathrm{~L}$ chemotherapy. In their study, poor Karnofsky performance status (less than 70\%) and a neutrophil-tolymphocyte ratio > 5 (NLR) were independent negative prognostic factors for a benefit of $2 \mathrm{~L}$ therapy. Tsang et al. (25) performed a multivariate analysis and found that a better ECOG score, locally advanced disease and $1 \mathrm{~L}$ treatment with FOLFIRINOX were the best prognostic factors for receiving a benefit from $2 \mathrm{~L}$ therapy. All of these proposed prognostic factors need to be validated in prospective studies. However, 
these studies provide a preliminary list of factors clinicians should pay attention to when deciding whether to administer $2 \mathrm{~L}$ treatment to patients.

PAC is unfortunately often diagnosed in a late stage, thus substantially narrowing the treatment options and leading to a poor outcome. There is an urgent need for new techniques and biomarkers to facilitate the diagnosis of PAC in an earlier stage and to facilitate therapeutic decision making and the determination of follow-up strategies. Liquid biopsies (LBs) are a promising non-invasive approach to the generation of personalized cancer treatment strategies. Improvement in LB techniques is urgently needed as is the standardization of LB protocols. However, these techniques are becoming widely recognized as fundamental tools in clinical practice, as highlighted in the meta-analysis conducted by Luchini et al. (44).

Unfortunately, long-term remission of PAC remains rare, even with the current standard treatments, namely, chemotherapy and surgery, and personalized medicine. This indicates the urgent need for new therapeutic and diagnostic strategies. To this end, the POLO RCT indicated that therapy with olaparib significantly delayed the progression of metastatic PAC with a BRCA germline mutation (45). While immunotherapy (IO) has been increasingly important in many other cancers, it has not been shown to be effective in PAC. Efforts have been made to broaden the impact of $I O$ in PAC, focusing on the stimulation of the anti-tumour T-cell response and the enhancement of tumour sensitivity to chemotherapy (46). Currently, however, it remains unclear whether concurrent chemotherapy and IO is a viable therapeutic approach. Preclinical studies have shown the potential success of this combination, but practical clinical evidence has not yet been obtained.

\section{Conclusion}

This retrospective RWD study strengthens the view that active treatment is worth pursuing for most patients with PAC. Our study shows the benefit of treating those patients who are able to tolerate treatment for longer periods of time. Our results also highlight the urgent need for novel therapies and diagnostic methods. Clear selection criteria for PC patients who should continue to receive active therapy should be further studied, especially in patients with ECOG scores of 2 who are older than 70 years to minimize fatal complications and preserve quality of life.

\section{Abbreviations}

Real-world data (RWD), Pancreatic cancer (PC), Nab-paclitaxel (nab-P), Pancreatic adenocarcinoma (PAC), Gemcitabine (Gem), Adverse effect (AE)

\section{Declarations}

\section{Ethics approval and consent to participate}


Permission to conduct this retrospective study and access patient records was obtained as needed by the chief physician and the Vaasa Central Hospital medical research ethics committee, thereby waiving the need for individual patient informed consent to be obtained.

\section{Consent for publication}

Not applicable.

\section{Availability of data and materials}

The datasets used and analyzed during the current study are available from the corresponding author upon reasonable request.

\section{Competing interests}

The authors declare that they have no competing interests.

\section{Funding}

The study was supported by Tampere EVO grant. The funding body had no role in the design, collection, analysis or interpretation of the data.

\section{Authors' contributions}

AJ, the principal investigator, conceived the study and supervised the project. GS and JV collected data, TV performed statistical analyses, JV and NE interpreted the data and drafted the manuscript, AJ reviewed manuscript. All authors read and approved the final version of the manuscript.

\section{Acknowledgements}

This article was checked for proper English language, grammar and spelling by the American Journal Experts (AJE).

\section{References}

1. Finnish cancer. statistics 2013-2017 [Cancer related deaths in Finland 2013-7]. Available from: https://cancerregistry.fi/statistics/cancer-statistics/.

2. Ferlay J, Soerjomataram I, Dikshit R, Eser S, Mathers C, Rebelo M, et al. Cancer incidence and mortality worldwide: Sources, methods and major patterns in GLOBOCAN 2012. Int J Cancer. 2015;136(5):E359-E86. 
3. Siegel RL, Miller KD, Jemal A. Cancer statistics. 2018. CA: A Cancer Journal for Clinicians. 2018;68(1):7-30.

4. Taieb J, Pointet AL, Van Laethem JL, Laquente B, Pernot S, Lordick F, et al. What treatment in 2017 for inoperable pancreatic cancers? Annals of oncology: official journal of the European Society for Medical Oncology. 2017;28(7):1473-83.

5. 3rd Moore HAB, Andersen MJ, Green J, Rothenberg MR, Modiano ML. MR, et al. Improvements in survival and clinical benefit with gemcitabine as first-line therapy for patients with advanced pancreas cancer: a randomized trial. J Clin Oncol. 1997;15(6):2403-13.

6. Hedy L, Kindler TI, Dirk J, Richel J, Bennouna R, Létourneau T, Okusaka A, Funakoshi J, Furuse YS, Park S, Ohkawa GM, Springett, Harpreet S, Wasan, Peter C, Trask P, Bycott, Alejandro D, Ricart. Sinil Kim, Eric Van Cutsem. Axitinib plus gemcitabine versus placebo plus gemcitabine in patients with advanced pancreatic adenocarcinoma: a double-blind randomised phase 3 study. The Lancet Oncology. 2011;12(3):256-62.

7. Herrmann R, Bodoky G, Ruhstaller T, Glimelius B, Bajetta E, Schüller J, et al. Gemcitabine Plus Capecitabine Compared With Gemcitabine Alone in Advanced Pancreatic Cancer: A Randomized, Multicenter, Phase III Trial of the Swiss Group for Clinical Cancer Research and the Central European Cooperative Oncology Group. J Clin Oncol. 2007;25(16):2212-7.

8. Louvet C, Labianca R, Hammel P, Lledo G, Zampino MG, André T, et al. Gemcitabine in Combination With Oxaliplatin Compared With Gemcitabine Alone in Locally Advanced or Metastatic Pancreatic Cancer: Results of a GERCOR and GISCAD Phase III Trial. J Clin Oncol. 2005;23(15):3509-16.

9. Moore MJ, Goldstein D, Hamm J, Figer A, Hecht JR, Gallinger S, et al. Erlotinib Plus Gemcitabine Compared With Gemcitabine Alone in Patients With Advanced Pancreatic Cancer: A Phase III Trial of the National Cancer Institute of Canada Clinical Trials Group. J Clin Oncol. 2007;25(15):1960-6.

10. Rocha Lima CM, Green MR, Rotche R, Miller WH, Jeffrey GM, Cisar LA, et al. Irinotecan Plus Gemcitabine Results in No Survival Advantage Compared With Gemcitabine Monotherapy in Patients With Locally Advanced or Metastatic Pancreatic Cancer Despite Increased Tumor Response Rate. J Clin Oncol. 2004;22(18):3776-83.

11. Van Cutsem E, Vervenne WL, Bennouna J, Humblet Y, Gill S, Van Laethem J-L, et al. Phase III Trial of Bevacizumab in Combination With Gemcitabine and Erlotinib in Patients With Metastatic Pancreatic Cancer. J Clin Oncol. 2009;27(13):2231-7.

12. Von Hoff DD, Ervin T, Arena FP, Chiorean EG, Infante J, Moore M, et al. Increased survival in pancreatic cancer with nab-paclitaxel plus gemcitabine. N Engl J Med. 2013;369(18):1691-703.

13. Conroy T, Desseigne F, Ychou M, Bouche O, Guimbaud R, Becouarn Y, et al. FOLFIRINOX versus gemcitabine for metastatic pancreatic cancer. N Engl J Med. 2011;364(19):1817-25.

14. Ellenrieder V, König A, Seufferlein T. Current Standard and Future Perspectives in First- and SecondLine Treatment of Metastatic Pancreatic Adenocarcinoma. Digestion. 2016;94(1):44-9.

15. Sohal DPS, Kennedy EB, Khorana A, Copur MS, Crane CH, Garrido-Laguna I, et al. Metastatic Pancreatic Cancer: ASCO Clinical Practice Guideline Update. J Clin Oncol. 2018;36(24):2545-56. 
16. Tempero MA, Malafa MP, Al-Hawary M, Asbun H, Bain A, Behrman SW, et al. Pancreatic Adenocarcinoma, Version 2.2017, NCCN Clinical Practice Guidelines in Oncology. J Natl Compr Canc Netw. 2017;15(8):1028-61.

17. Abrams TA, Meyer G, Meyerhardt JA, Wolpin BM, Schrag D, Fuchs CS. Patterns of Chemotherapy Use in a U.S.-Based Cohort of Patients with Metastatic Pancreatic Cancer. Oncologist. 2017;22(8):92533.

18. Aroldi F, Bertocchi P, Savelli G, Rosso E, Zaniboni A. Pancreatic cancer: New hopes after first line treatment. World J Gastrointest Oncol. 2016;8(9):682-7.

19. Chiorean EG, Von Hoff DD, Tabernero J, El-Maraghi R, Ma WW, Reni M, et al. Second-line therapy after nab-paclitaxel plus gemcitabine or after gemcitabine for patients with metastatic pancreatic cancer. British journal of cancer. 2016;115(2):188-94.

20. Ducreux M, Seufferlein T, Van Laethem JL, Laurent-Puig P, Smolenschi C, Malka D, et al. Systemic treatment of pancreatic cancer revisited. Seminars in oncology. 2019;46(1):28-38.

21. Gill S, Ko Y-J, Cripps C, Beaudoin A, Dhesy-Thind S, Zulfiqar M, et al. PANCREOX: A Randomized Phase III Study of Fluorouracil/Leucovorin With or Without Oxaliplatin for Second-Line Advanced Pancreatic Cancer in Patients Who Have Received Gemcitabine-Based Chemotherapy. J Clin Oncol. 2016;34(32):3914-20.

22. Oettle H, Riess H, Stieler JM, Heil G, Schwaner I, Seraphin J, et al. Second-Line Oxaliplatin, Folinic Acid, and Fluorouracil Versus Folinic Acid and Fluorouracil Alone for Gemcitabine-Refractory Pancreatic Cancer: Outcomes From the CONKO-003 Trial. 2014;32(23):2423-9.

23. Pokataev I, Bazin I, Fedyanin M, Tryakin A, Popova A, Rumyantsev A, et al. Clinical predictors of second-line chemotherapy (ChT) benefit in pancreatic cancer (PC). J Clin Oncol. 2017;35(15_suppl):e15733-e.

24. Sinn M, Dälken L, Striefler JK, Bischoff S, Schweitzer N, Pelzer U, et al. Second-Line Treatment in Pancreatic Cancer Patients: Who Profits?-Results From the CONKO Study Group. Pancreas. 2016;45(4):601-5.

25. Tsang ES, Wong HL, Wang Y, Renouf DJ, Cheung WY, Lim HJ, et al. Outcomes and Characteristics of Patients Receiving Second-line Therapy for Advanced Pancreatic Cancer. Am J Clin Oncol. 2019;42(2):196-201.

26. Vienot A, Beinse G, Louvet C, De Mestier L, Meurisse A, Fein F, et al. Overall Survival Prediction and Usefulness of Second-Line Chemotherapy in Advanced Pancreatic Adenocarcinoma. JNCl: Journal of the National Cancer Institute. 2017;109(10).

27. Wang-Gillam A, Li C-P, Bodoky G, Dean A, Shan Y-S, Jameson G, et al. Nanoliposomal irinotecan with fluorouracil and folinic acid in metastatic pancreatic cancer after previous gemcitabine-based therapy (NAPOLI-1): a global, randomised, open-label, phase 3 trial. The Lancet. 2016;387(10018):545-57.

28. Wang Y, Camateros P, Cheung WY. A Real-World Comparison of FOLFIRINOX, Gemcitabine Plus nabPaclitaxel, and Gemcitabine in Advanced Pancreatic Cancers. Journal of Gastrointestinal Cancer. 
2019;50(1):62-8.

29. Blomstrand H, Scheibling U, Bratthäll C, Green H, Elander NO. Real world evidence on gemcitabine and nab-paclitaxel combination chemotherapy in advanced pancreatic cancer. BMC cancer. 2019;19(1).

30. Ali A, Kanth A, Mohanty R, Gowda A, Patil A. Real World Evidence: An Overview of its Importance in the Current Scenario2016. 251-4 p.

31. Graham S, McDonald L, Wasiak R, Lees M, Ramagopalan S. Time to really share real-world data? F1000Res. 2018;7:1054-.

32. Katkade VB, Sanders KN, Zou KH. Real world data: an opportunity to supplement existing evidence for the use of long-established medicines in health care decision making. Journal of multidisciplinary healthcare. 2018;11:295-304.

33. Kalra D. The importance of real-world data to precision medicine. Personalized Medicine. 2019.

34. Dimitrakopoulos C, Vrugt B, Flury R, Schraml P, Knippschild U, Wild P, et al. Identification and Validation of a Biomarker Signature in Patients With Resectable Pancreatic Cancer via Genome-Wide Screening for Functional Genetic VariantsA Biomarker Signature in Resectable Pancreatic Cancer via Genome-Wide Screening for Functional Genetic VariantsA Biomarker Signature in Resectable Pancreatic Cancer via Genome-Wide Screening for Functional Genetic Variants. JAMA Surgery. 2019;154(6):e190484-e.

35. Lupinacci RM, Goloudina A, Buhard O, Bachet JB, Marechal R, Demetter P, et al. Prevalence of Microsatellite Instability in Intraductal Papillary Mucinous Neoplasms of the Pancreas. Gastroenterology. 2018;154(4):1061-5.

36. Li W, Tao L, Lu M, Xiu D. Prognostic role of platelet to lymphocyte ratio in pancreatic cancers: A metaanalysis including 3028 patients. Medicine. 2018;97(8):e9616.

37. Qian L, Yu S, Yin C, Zhu B, Chen Z, Meng Z, et al. Plasma IFN-y-inducible chemokines CXCL9 and CXCL10 correlate with survival and chemotherapeutic efficacy in advanced pancreatic ductal adenocarcinoma. Pancreatology. 2019;19(2):340-5.

38. Zhang Q, Chen S, Zeng L, Chen Y, Lian G, Qian C, et al. New developments in the early diagnosis of pancreatic cancer. Expert Review of Gastroenterology Hepatology. 2017;11(2):149-56.

39. De Vita F, Ventriglia J, Febbraro A, Laterza MM, Fabozzi A, Savastano B, et al. NAB-paclitaxel and gemcitabine in metastatic pancreatic ductal adenocarcinoma (PDAC): from clinical trials to clinical practice. BMC Cancer. 2016;16(1):709.

40. Kim G. nab-Paclitaxel for the treatment of pancreatic cancer. Cancer management research. 2017:9:85-96.

41. Braiteh F, Patel MB, Parisi M, Ni Q, Park S, Faria C. Comparative effectiveness and resource utilization of nab-paclitaxel plus gemcitabine vs FOLFIRINOX or gemcitabine for the first-line treatment of metastatic pancreatic adenocarcinoma in a US community setting. Cancer management research. 2017;9:141-8. 
42. Montes A, Villarroel P, Ayerbes M, \#243, mez J, Aldana G, et al. Prognostic and predictive markers of response to treatment in patients with locally advanced unresectable and metastatic pancreatic adenocarcinoma treated with gemcitabine/nab-paclitaxel: Results of a retrospective analysis. J Cancer Res Ther. 2017;13(2):240-5.

43. Sasaki T, Kanata R, Yamada I, Matsuyama M, Ozaka M, Sasahira N. Improvement of Treatment Outcomes for Metastatic Pancreatic Cancer: A Real-world Data Analysis. In vivo. 2019;33(1):271-6.

44. Luchini C, Veronese N, Nottegar A, Cappelletti V, Daidone MG, Smith L, et al. Liquid Biopsy as Surrogate for Tissue for Molecular Profiling in Pancreatic Cancer: A Meta-Analysis Towards Precision Medicine. Cancers. 2019;11(8).

45. Golan T, Hammel P, Reni M, Van Cutsem E, Macarulla T, Hall MJ, et al. Maintenance Olaparib for Germline BRCA-Mutated Metastatic Pancreatic Cancer. N Engl J Med. 2019;381(4):317-27.

46. Balachandran VP, Beatty GL, Dougan SK. Broadening the Impact of Immunotherapy to Pancreatic Cancer: Challenges and Opportunities. Gastroenterology. 2019;156(7):2056-72.

\section{Tables}

\section{Table 1. Baseline characteristics of patients diagnosed with PAC}

This table shows the baseline characteristics of patients included in the study. The table is divided by stage of disease and sex. The variables included are to the left of the table. The far-right column works as a summary. 


\begin{tabular}{|c|c|c|c|c|c|c|c|}
\hline & \multicolumn{2}{|c|}{ Locally Advanced } & \multicolumn{2}{|c|}{ Metastatic Disease } & \multicolumn{2}{|c|}{ Resectable } & \multirow{2}{*}{ Sum } \\
\hline & Female & Male & Female & Male & Female & Male & \\
\hline$N$ & 27 & 27 & 35 & 38 & 9 & 12 & 148 \\
\hline Age & $71.6(67.3,75.9)$ & $69.6(65.9,73.2)$ & $72.8(69.5,76.2)$ & $69(66.1,71.9)$ & $69.2(65.5,72.9)$ & $68.4(64.2,72.5)$ & $70.5(68.9,72)$ \\
\hline$<50$ & 2 & 0 & 0 & 1 & 0 & 1 & 4 \\
\hline $50-70$ & 12 & 13 & 15 & 21 & 6 & 4 & 71 \\
\hline$>70$ & 13 & 14 & 20 & 16 & 3 & 7 & 73 \\
\hline \multicolumn{8}{|l|}{$\begin{array}{l}\text { ECOG ps } \\
(1-3):\end{array}$} \\
\hline ps1 & 5 & 5 & 5 & 4 & 0 & 3 & 22 \\
\hline ps2 & 19 & 20 & 22 & 27 & 9 & 9 & 106 \\
\hline ps3 & 3 & 2 & 8 & 7 & 0 & 0 & 20 \\
\hline $\begin{array}{l}\text { P-ALAT } \\
(\mathrm{U} / 1)\end{array}$ & $27(13,540)$ & $31(12,393)$ & $32(11,726)$ & $30(6.9,329)$ & $45(16,891)$ & $38.5(14,530)$ & $31(6.9,891)$ \\
\hline $\begin{array}{l}\text { P-Bilirubin } \\
\text { (umol/l) }\end{array}$ & $13(6,122)$ & $16(4,370)$ & $13(3,376)$ & $12(3,682)$ & $13(5,95)$ & $15.5(3,162)$ & $13(3,682)$ \\
\hline $\begin{array}{l}\text { S-Ca19-9 } \\
(\mathrm{kU} / \mathrm{l})\end{array}$ & $49(2,14124)$ & $176(2,10685)$ & $2194(6,8478400)$ & $1211.5(4,871770)$ & $22(3,536)$ & $15(3,308)$ & $284(2,8478400)$ \\
\hline Diabetes & 7 & 7 & 13 & 16 & 5 & 4 & 52 \\
\hline Opioids & 21 & 15 & 18 & 28 & 6 & 6 & 94 \\
\hline $\begin{array}{l}\text { Adjuvant } \\
\text { therapy } \\
\text { for } \\
\text { pancreatic } \\
\text { cancer }\end{array}$ & 2 & 2 & 0 & 2 & 8 & 10 & 24 \\
\hline $\begin{array}{l}\text { Primary } \\
\text { tumor size } \\
(\mathrm{cm})\end{array}$ & $3.5(0.4,7.5)$ & $4(2.5,14)$ & $3.4(1.2,6.6)$ & $4.5(1,8)$ & $3.2(2.5 .4)$ & $3.5(1.6,5.5)$ & $3.7(0.4,14)$ \\
\hline \multicolumn{8}{|l|}{$\begin{array}{l}\text { Primary } \\
\text { tumor } \\
\text { location: }\end{array}$} \\
\hline Head & 16 & 15 & 18 & 23 & 9 & 10 & 91 \\
\hline Body & 2 & 2 & 6 & 3 & 0 & 0 & 13 \\
\hline Other & 8 & 9 & 8 & 8 & 0 & 2 & 35 \\
\hline $\begin{array}{l}\text { Secondary } \\
\text { cancer }\end{array}$ & 4 & 1 & 0 & 2 & 2 & 0 & 9 \\
\hline $\begin{array}{l}\text { Major } \\
\text { comorbid } \\
\text { illness }\end{array}$ & 2 & 7 & 7 & 5 & 1 & 4 & 26 \\
\hline
\end{tabular}

\section{Table 2. Adverse effects (AEs) recorded during PAC treatment}

In this table, all the side effects are divided in two ways. First, they are divided by the grade of the effect. Second, they are divided by the tumour stage at diagnosis. In the summary column, you can see the total number of side effects. This table does not take into account the number of patients. Some of the patients may have had many side effects, and some of them may not have any side effects. The remainder of the table is presented on the following page. 


\begin{tabular}{|c|c|c|c|c|c|c|c|c|c|}
\hline \multirow{2}{*}{ Adverse Effects } & \multicolumn{5}{|c|}{ Grade } & \multicolumn{3}{|c|}{ Tumor stage at diagnosis } & \multirow{2}{*}{ - Sum } \\
\hline & 1 & 2 & 3 & 4 & 5 & Locally Advanced & Metastatic Disease & Resectable & \\
\hline anemia & 0 & 14 & 5 & 0 & 0 & 12 & 6 & 1 & 19 \\
\hline ascites & 0 & 0 & 3 & 0 & 0 & 0 & 3 & 0 & 3 \\
\hline biliary stasis & 0 & 0 & 4 & 0 & 0 & 1 & 3 & 0 & 4 \\
\hline biliary stent obstruction & 0 & 0 & 1 & 0 & 0 & 0 & 0 & 1 & 1 \\
\hline cholecystitis & 0 & 0 & 2 & 0 & 1 & 1 & 1 & 1 & 3 \\
\hline constipation & 1 & 1 & 1 & 0 & 0 & 1 & 2 & 0 & 3 \\
\hline diabetes mellitus & 0 & 1 & 0 & 0 & 0 & 0 & 1 & 0 & 1 \\
\hline diarrhoea & 4 & 2 & 7 & 0 & 1 & 4 & 4 & 6 & 14 \\
\hline dyspepsia & 0 & 2 & 0 & 0 & 0 & 1 & 1 & 0 & 2 \\
\hline edema & 0 & 1 & 0 & 0 & 0 & 0 & 1 & 0 & 1 \\
\hline fatigue & 4 & 3 & 0 & 0 & 0 & 0 & 1 & 6 & 7 \\
\hline febrile neutropenia & 0 & 0 & 3 & 2 & 2 & 3 & 2 & 2 & 7 \\
\hline fever & 1 & 0 & 1 & 0 & 0 & 3 & 1 & 0 & 4 \\
\hline Gl hemorrhage & 0 & 0 & 1 & 0 & 2 & 1 & 1 & 1 & 3 \\
\hline hand-foot syndrome & 1 & 1 & 1 & 0 & 0 & 0 & 2 & 1 & 3 \\
\hline hemorrhage & 0 & 0 & 1 & 0 & 1 & 1 & 1 & 0 & 2 \\
\hline infection & 0 & 1 & 5 & 0 & 0 & 2 & 6 & 0 & 8 \\
\hline insomnia & 0 & 0 & 1 & 0 & 0 & 0 & 1 & 0 & 1 \\
\hline kidney failure & 0 & 1 & 0 & 0 & 0 & 1 & 0 & 0 & 1 \\
\hline leukopenia & 1 & 29 & 20 & 0 & 1 & 16 & 25 & 10 & 51 \\
\hline nausea & 0 & 3 & 8 & 0 & 0 & 5 & 3 & 3 & 11 \\
\hline neuropathy & 1 & 1 & 1 & 0 & 0 & 1 & 2 & 0 & 3 \\
\hline neutropenia & 24 & 41 & 8 & 6 & 0 & 35 & 26 & 18 & 79 \\
\hline others & 1 & 0 & 0 & 0 & 0 & 5 & 8 & 1 & 14 \\
\hline pneumonia & 0 & 0 & 7 & 0 & 0 & 2 & 4 & 1 & 7 \\
\hline portal thrombosis & 0 & 0 & 1 & 0 & 0 & 0 & 0 & 1 & 1 \\
\hline portal vein thrombosis & 0 & 0 & 1 & 0 & 0 & 1 & 0 & 0 & 1 \\
\hline pulmonary embolism & 0 & 0 & 0 & 1 & 0 & 1 & 0 & 0 & 1 \\
\hline pulmonary thromboembolism & 0 & 0 & 1 & 1 & 0 & 0 & 2 & 0 & 2 \\
\hline sepsis & 0 & 0 & 0 & 2 & 3 & 3 & 1 & 1 & 5 \\
\hline severe infection & 0 & 0 & 0 & 2 & 0 & 1 & 1 & 0 & 2 \\
\hline thrombocytopenia & 1 & 5 & 4 & 0 & 1 & 8 & 2 & 1 & 11 \\
\hline venous thromboembolism & 0 & 4 & 0 & 0 & 0 & 0 & 4 & 0 & 4 \\
\hline vertigo & 0 & 1 & 0 & 0 & 0 & 0 & 1 & 0 & 1 \\
\hline vomiting & 0 & 0 & 1 & 0 & 0 & 1 & 0 & 0 & 1 \\
\hline
\end{tabular}

Figures 
Figure 1.
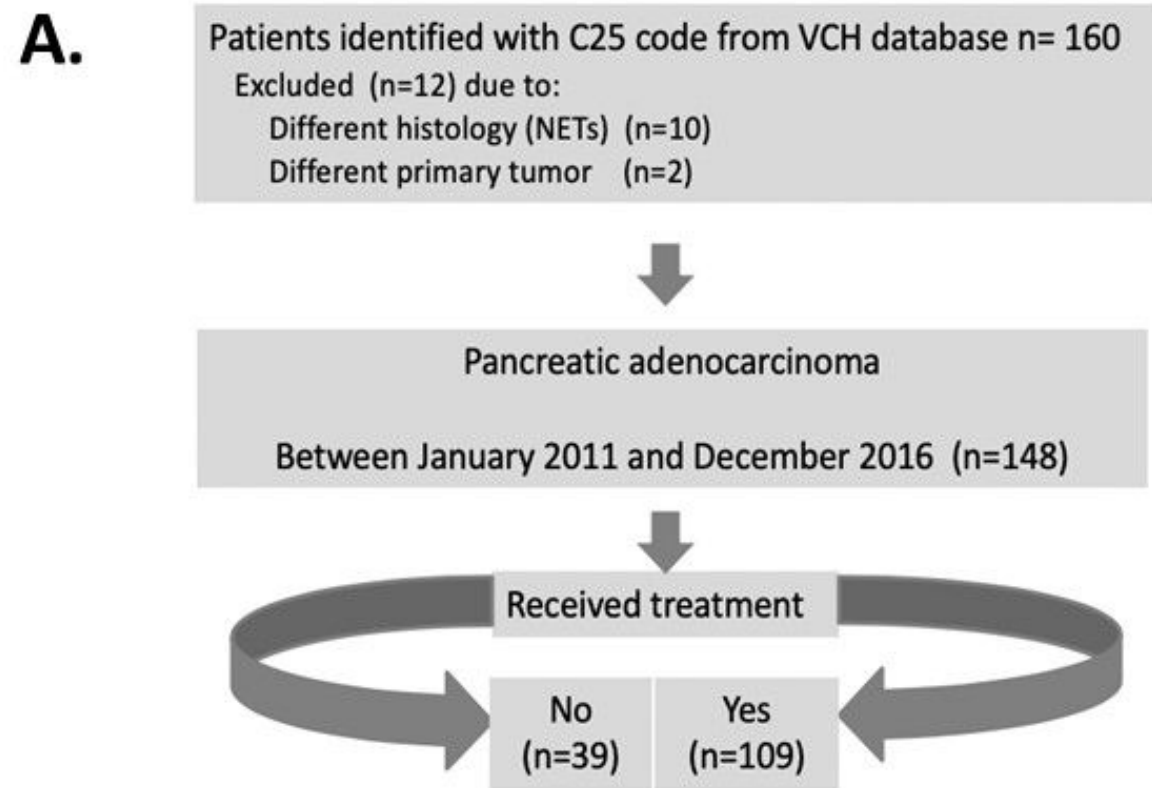

B.

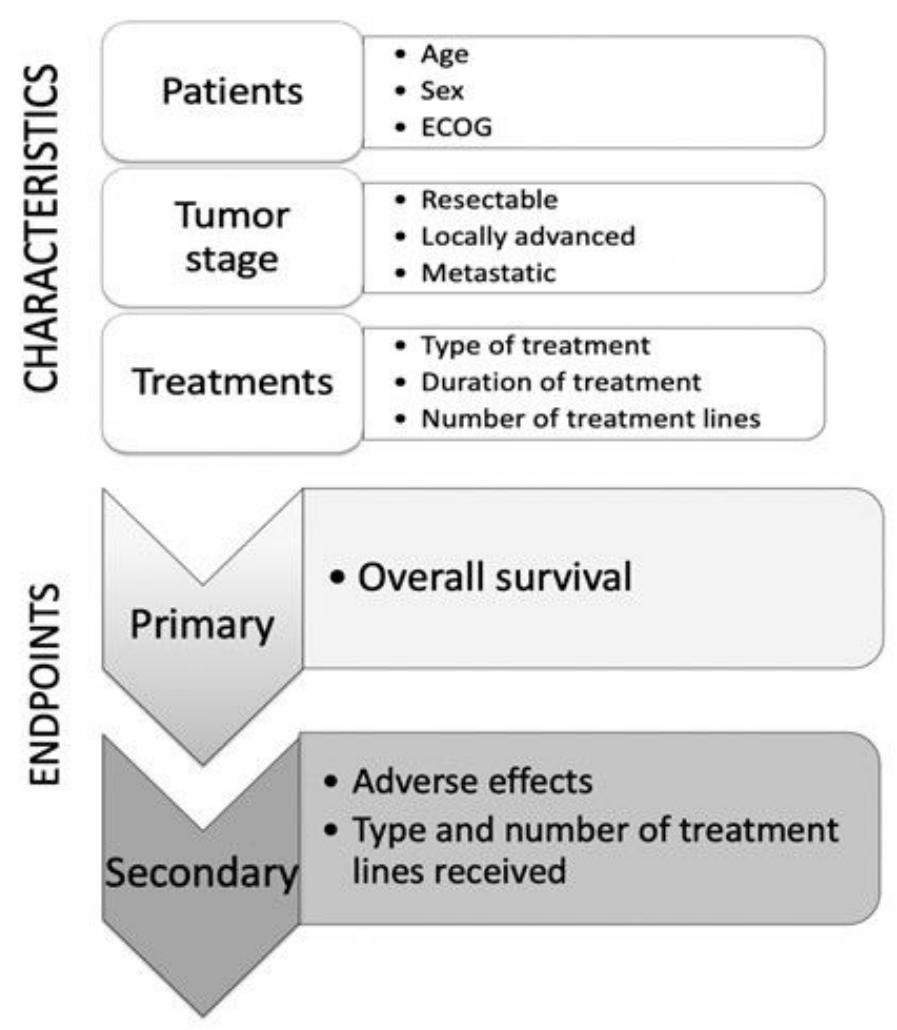

\section{Figure 1}

Search criteria and aims of the study A. This figure shows the search criteria and relevant factors for inclusion to the study, as well as a flow-chart and exclusion numbers. B. This figure shows the aims of the study, all researched variables as well as primary and secondary endpoints. 
Figure 2.
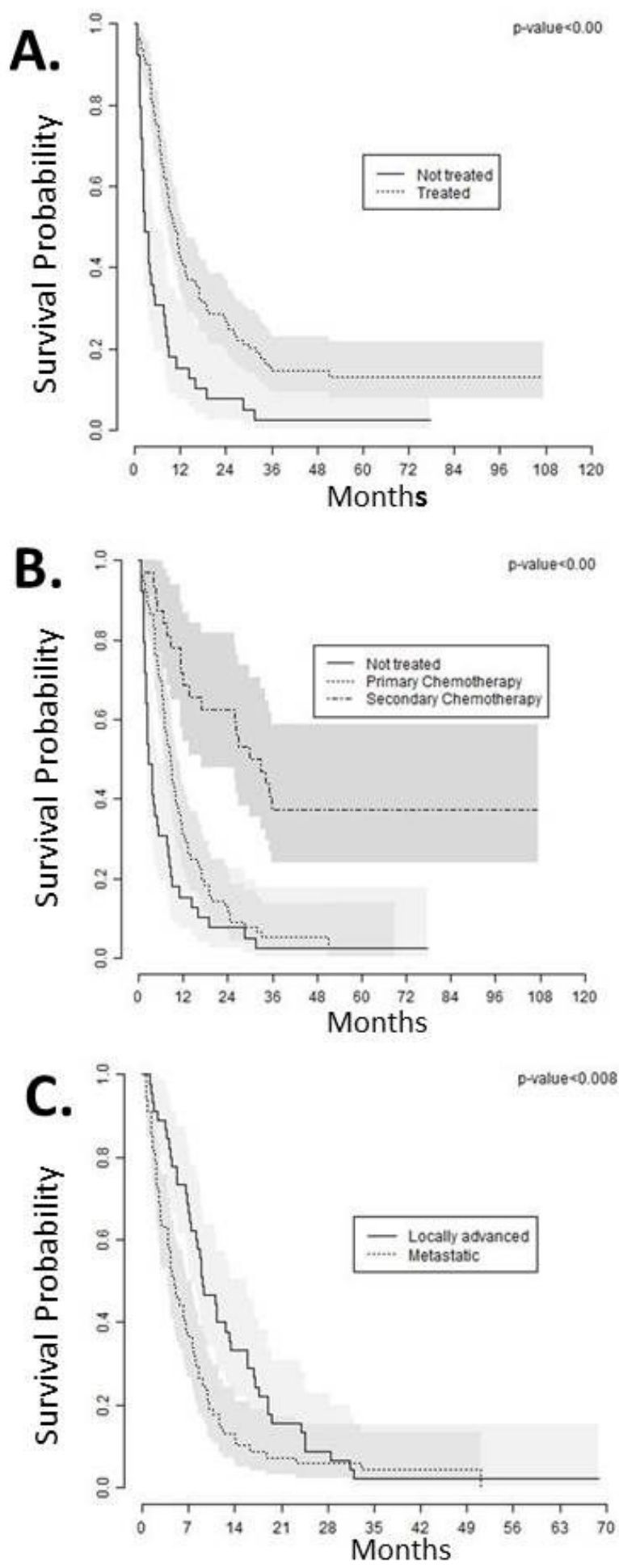

Figure 2

Survival according to treatment and stage A. Survival in the untreated and treated groups. This figure displays the overall survival (OS) between patients who received treatment of any kind versus those patients who received no treatment at all. B. Survival in groups receiving no treatment, chemotherapy secondary to surgical intervention and chemotherapy as the primary form of treatment. This figure shows the overall survival between patients who underwent surgery, received primary treatment chemotherapy 
and those who received no treatment at all. C. Survival in patients with metastatic vs. locally advanced pancreatic cancer. This figure shows the overall survival between patients diagnosed with metastatic disease and locally advanced disease regardless of treatment received.

\section{Figure 3.}
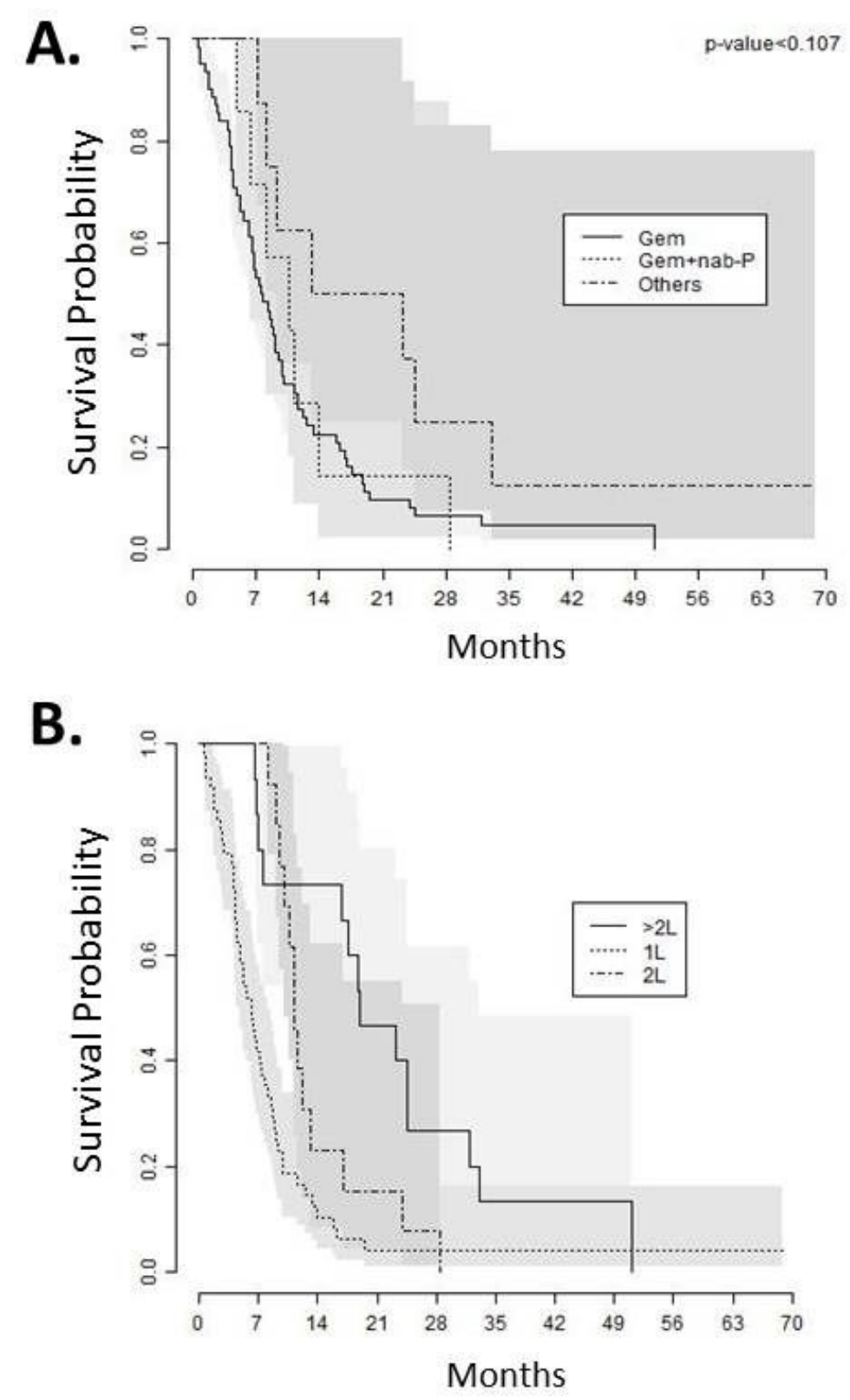

Figure 3

Survival according to regimens and lines of chemotherapy A. Survival according to first-line (1L) chemotherapy regimens in all treated patients. This figure displays the overall survival according to which 
regimen were given as first-line $(1 \mathrm{~L})$ treatment in patients who received chemotherapy as primary form of treatment. B. Survival according to the number of lines of chemotherapy. This figure displays the overall survival for patients according to how many treatment lines were given in total.

\section{Figure 4.}
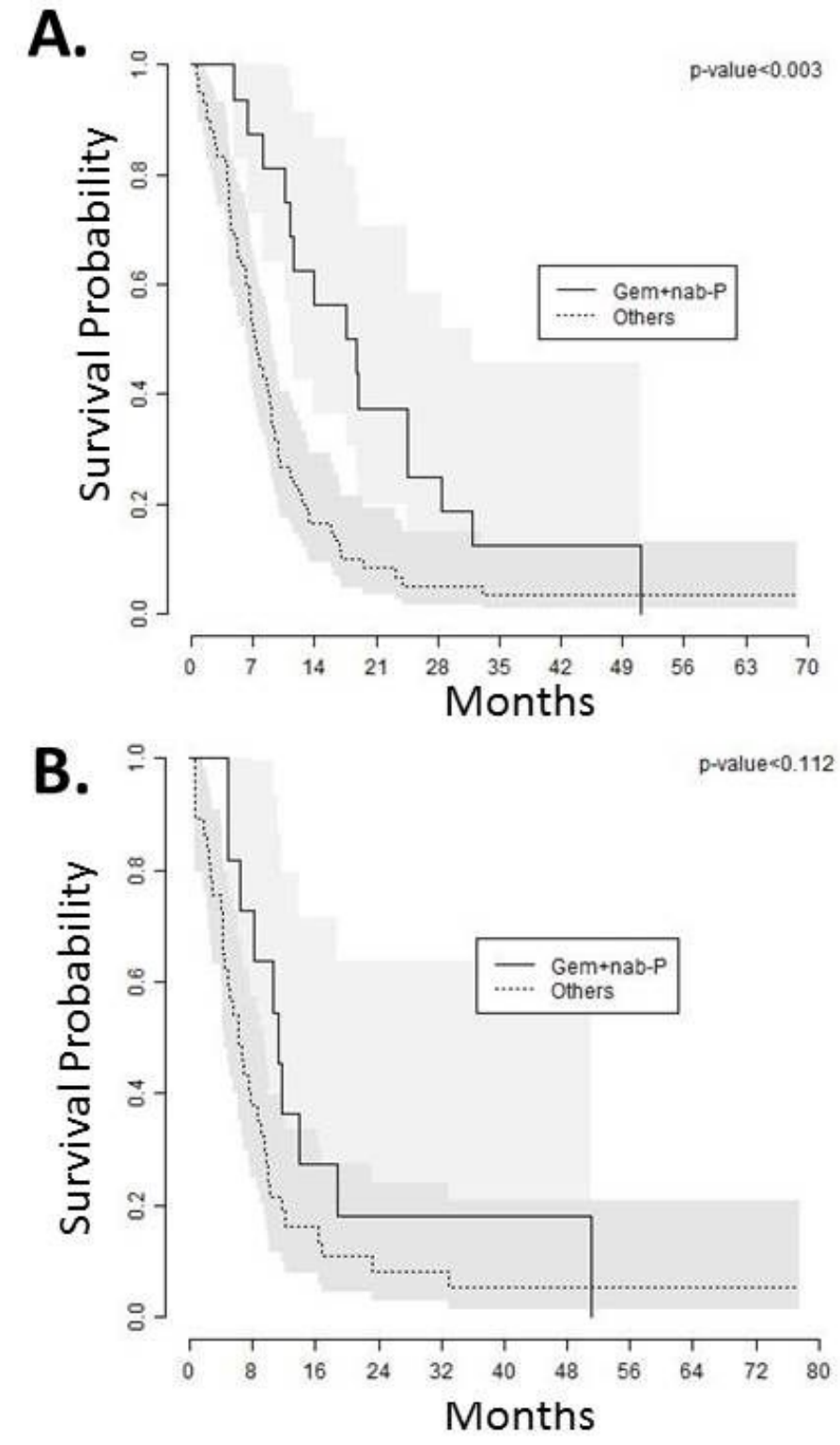

Figure 4

Comparison of overall survival with gem+nab-P vs other treatments according to stage of disease A. OS in those who received gem+nab-P in comparison with other treatments (all patients). This figure shows 
the overall survival for patients who received Gemcitabine and nab-Paclitaxel combination treatment in comparison with patients who received other treatment regimens. B. OS in those who received gem+nab$P$ in comparison with other treatments (patients with metastatic disease). This figure shows the overall survival for patients with metastatic cancer who received Gemcitabine and nab-Paclitaxel in comparison with other regimens.

\section{Supplementary Files}

This is a list of supplementary files associated with this preprint. Click to download.

- SupplementaryFigurelegends.docx

- Suppl.Figure2..jpg

- Suppl.Figure1..jpg 